\title{
Magnetic properties, interlayer exchange coupling and electric transport in $\mathrm{Fe} / \mathrm{Cr} / \mathrm{Fe}$ trilayers
}

\author{
A. Vernes $\dagger$ ๆ, P. Weinberger $\dagger$, C. Blaas $\dagger$, P. Mohn $\dagger$, L. Szunyogh $\dagger$, \\ P. M. Levy§ and C. Sommers $\|$ \\ $\dagger$ Center for Computational Materials Science, Gumpendorferstrase 1a, \\ A-1060 Vienna, Austria \\ $\ddagger$ Department of Theoretical Physics, Budapest University of Technology and \\ Economics, Budafoki út 8, H-1521, Budapest, Hungary \\ $\S$ Department of Physics, New York University, 4 Washington Place, New York, \\ New York 10003, USA \\ || Laboratoire de Physique des Solides, Université de Paris-Sud, 91405 Orsay, \\ France
}

[Received 27 April 2001 and accepted 4 May 2001]

\begin{abstract}
Based on the fully relativistic spin-polarized screened Korringa-KohnRostoker method and the Kubo-Greenwood equation as formulated for layered systems the interlayer exchange coupling (IEC) as well as the magnetoresistance (MR) for the current in-plane (CIP) geometry is calculated for $\mathrm{Fe} / \mathrm{Cr}$ / Fe trilayer systems. For the IEC not only short periods of 2 monolayers (ML) but also long periods of about $18 \mathrm{ML}$ are found. For large $\mathrm{Cr}$ thicknesses the magnetic moments oscillate with a period of 8-9 ML for even numbers of $\mathrm{Cr}$ layers and 18 for odd. The calculated CIP MR and corresponding resistivities are in the range of available experimental data. It is found that the peaks in the CIP MR have little in common with the oscillation periods characterizing the IEC.
\end{abstract}

\section{$\S 1$. INTRODUCTION}

$\mathrm{Fe} / \mathrm{Cr}$ multilayer systems have attached much experimental (Baibich et al. 1988, Krebs et al. 1989, Barnas et al. 1990, Barthélémy et al. 1990, Parkin et al. 1990, Baumgart et al. 1991, Chaiken et al. 1991, Krebs et al. 1991, Petroff et al. 1991, Purcell et al. 1991, Unguris et al. 1991, Fullerton et al. 1992, Gijs and Okada 1992a,b, 1993, Jacob et al. 1992, Kamijo and Igarashi 1992, Miyazaki et al. 1992, Obi et al. 1992, Gijs et al. 1993, 1994, Hosoito et al. 1993, Omi et al. 1993, Parkin and York 1993, Hahn et al. 1994, Potter et al. 1994, Schad et al. 1994a,b, 1995, 1996a,b, 1998, Colino et al. 1996, Ho and Petford-Long 1996, Rensing et al. 1996, Ueda et al. 1996, Ustinov et al. 1996a,b, Heinrich et al. 1999, 2000, Ito et al. 1999, Pierce et al. 1999) and theoretical interest (Levy et al. 1990, Levy 1991, Zhang et al. 1992, Stoeffler and Gautier 1993, Mertig et al. 1995, Mirbt et al. 1996, Tsymbal and Pettifor 1996, Mathon 1997, Fishman 1998, 1999, Costa et al. 1999, Fishman and Shi 1999, Hirai 1999, Koelling 1999, Niklasson et al. 1999, Hafner et al. 2000) which still

\footnotetext{
TEmail: av@cms.tuwien.ac.at

Philosophical Magazine B ISSN 1364-2812 print/ISSN 1463-6417 online (C) 2002 Taylor \& Francis Ltd 
has not yet ceased. It was essentially $\mathrm{Fe} / \mathrm{Cr} / \mathrm{Fe}$ trilayers or $\mathrm{Fe} / \mathrm{Cr}$ multilayers that lead to a first sizeable giant magnetoresistance (GMR) and showed oscillations in the so-called interlayer exchange coupling (IEC). Quite clearly over the past 10 years the experimental techniques have become much more sophisticated: variations in system parameters (thickness studies, etc.) and an increasingly careful recording of the growth conditions have given some insight into the conditions causing the GMR. Over the years, mostly so-called current-in-plane (CIP) measurements were carried out, although a magnetoresistance (MR) for a current perpendicular to the planes (CPP) geometry has been obtained (Gijs et al. 1994). Since some experimental measurements of interlayer coupling showed oscillations that coincide with peaks in the GMR, both effects were assumed to have the same physical origin. Very often the importance of studies of the IEC in trilayer systems were justified by referring to this correspondence with GMR. The discovery (Unguis et al. 1991) of the so-called long periods in the IEC with respect to the number of $\mathrm{Cr}$ layers invoked interpretations of this phenomenon in terms of spin-density waves and related properties of bulk $\mathrm{Cr}$.

In the present paper a unified fully relativistic spin-polarized approach to the IEC and electric transport in $\mathrm{Fe} / \mathrm{Cr} / \mathrm{Fe}$ trilayers is given. In order to reproduce the results of experiments on wedge-shaped samples the top layer of $\mathrm{Fe} / \mathrm{Cr} / \mathrm{Fe}$ trilayers on a bcc $\mathrm{Fe}(100)$ substrate is considered to be a free surface. All properties investigated are calculated using the same numerical methods.

In the following, first the theoretical concepts and the computational details are summarized. Then the results of the investigations performed are presented in the subsequent section, which in turn is followed by a comparison with experimental data and other theoretical approaches. We conclude by reviewing remaining problems and open questions.

\section{§2. Method of Calculation and computational Details}

\subsection{Self-consistent calculations}

The fully relativistic spin-polarized screened Korringa-Kohn-Rostoker method for layered systems (Szunyogh et al. 1995, Weinberger and Szunyogh 2000) is applied to calculate the electronic structure and magnetic properties of free surfaces of $\mathrm{Fe} /$ $\mathrm{Cr} / \mathrm{Fe}$ trilayers on $\mathrm{Fe}$ with growth direction along (100). In particular, systems of the type bcc $\mathrm{Fe}(100) / \mathrm{Fe}_{6} \mathrm{Cr}_{n} \mathrm{Fe}_{6} /$ vacuum, $1 \leqslant n \leqslant 45$, were investigated with six layers of $\mathrm{Fe}$ serving as a buffer to the substrate (left) and six Fe layers as cap (right). In all calculations a bcc Fe parent lattice (Weinberger 1997) is assumed with a lattice spacing of $5.27 \mathrm{au}$ (bulk bcc Fe), that is no layer relaxation is considered. In order to determine self-consistently within the local-density approximation (LDA) (Vosko et al. 1980) the effective potentials and effective exchange fields for each particular system under consideration, a minimum of $45 \mathbf{k}_{\|}$points in the irreducible wedge of the surface Brillouin zone (ISBZ) was used. Unless specified otherwise, all self-consistent calculations refer to a ferromagnetic configuration with the orientation of the magnetization parallel to the surface normal (the configuration $\mathcal{C}_{0}$ in table 1).

\subsection{Interlayer exchange coupling}

The interlayer coupling energy corresponds to the (total) energy difference between two given magnetic configurations:

$$
\Delta E=E(\mathcal{C})-E\left(\mathcal{C}_{0}\right),
$$


where $\mathcal{C}_{0}$ usually is termed the magnetic reference configuration (e.g. a ferromagnetic alignment in the case of IEC) and $\mathcal{C}$ is a magnetic configuration different from $\mathcal{C}_{0}$, for example a particular antiferromagnetic alignment. Here this energy difference is evaluated by making use of the magnetic force theorem which implies that only the reference configuration is determined self-consistently within the local-densityfunctional approximation and $\Delta E$ is replaced by the respective difference in the grand canonical potentials:

$$
\begin{gathered}
\Delta E \sim \Delta E_{\mathrm{b}}=\sum_{p=1}^{n} \Delta E_{\mathrm{b}}^{p}, \\
\Delta E_{\mathrm{b}}^{p}=\int_{\epsilon_{b}}^{\epsilon_{\mathrm{F}}}\left[n^{p}(\epsilon ; \mathcal{C})-n^{p}\left(\epsilon ; \mathcal{C}_{0}\right)\right]\left(\epsilon-\epsilon_{\mathrm{F}}\right) \mathrm{d} \epsilon,
\end{gathered}
$$

which as indicated in equation (3) can be written in terms of layer-dependent quantities $\Delta E_{\mathrm{b}}^{p}$ with $n$ denoting the total number of layers $p, n^{p}(\epsilon ; \mathcal{C})$ layer-resolved densities of states for a given magnetic configuration $\mathcal{C}, \epsilon_{\mathrm{b}}$ the (valence-)band bottom and $\epsilon_{\mathrm{F}}$ the Fermi energy of the substrate. It should be noted that, according to equation (1) $\Delta E_{\mathrm{b}}>0$ implies that $\mathcal{C}_{0}$ is the energetically preferred magnetic configuration. In the present paper, all $\Delta E_{\mathrm{b}}$ are evaluated by using a total of $990 \mathbf{k}_{\|}$points in the ISBZ which, as was shown (Weinberger and Szunyogh 2000) in the case of magnetic anisotropy energies, guarantees well-converged results.

\subsection{Electric transport}

For a given magnetic configuration $\mathcal{C}_{i}$ and number of atomic planes $n$ the inplane (CIP) conductivity is given by (Weinberger et al. 1996, Blaas et al. 1999, 2001)

$$
\sigma_{x x}\left(\mathcal{C}_{i} ; n\right)=\lim _{\delta \rightarrow 0}\left[\sigma_{x x}\left(\mathcal{C}_{i} ; n ; \delta\right)\right]
$$

where

$$
\sigma_{x x}\left(\mathcal{C}_{i} ; n ; \delta\right)=\sum_{i, j=1}^{n} \sigma_{x x}^{i j}\left(\mathcal{C}_{i} ; n ; \delta\right),
$$

and $\delta$ denotes the imaginary part of the complex Fermi energy $\epsilon_{\mathrm{F}}+\mathrm{i} \delta$. The corresponding resistivity is then defined by

$$
\rho_{\mathrm{CIP}}\left(\mathcal{C}_{i} ; n\right) \equiv \lim _{\delta \rightarrow 0}\left[\rho_{x x}\left(\mathcal{C}_{i} ; n ; \delta\right)\right]=\lim _{\delta \rightarrow 0}\left[\sigma_{x x}\left(\mathcal{C}_{i} ; n ; \delta\right)^{-1}\right]
$$

and the MR by

$$
R=\frac{\rho_{\mathrm{CIP}}(\mathcal{A P} ; n)-\rho_{\mathrm{CIP}}(\mathcal{P} ; n)}{\rho_{\mathrm{CIP}}(\mathcal{A P} ; n)},
$$

where $\mathcal{A P}$ and $\mathcal{P}$ denote the parallel (ferromagnetic) and antiparallel (antiferromagnetic) configurations, respectively. In this study, $R$ is approximated by $R(\delta)$ :

$$
R(\delta)=\frac{\rho_{\mathrm{CIP}}(\mathcal{A P} ; n ; \delta)-\rho_{\mathrm{CIP}}(\mathcal{P} ; n ; \delta)}{\rho_{\mathrm{CIP}}(\mathcal{A P} ; n ; \delta)} ;
$$

however, one has to keep in mind that $R \geqslant R(\delta)$.

All electric transport calculations were performed applying the fully relativistic spin-polarized form of the Kubo-Greenwood equation for layered systems 
Table 1. Magnetic configurations of $\mathrm{Fe} / \mathrm{Cr}_{n} / \mathrm{Fe}$ trilayers where $\hat{\mathbf{x}}$ and $\hat{\mathbf{y}}$ are unit vectors in the planes of atoms, $\hat{\mathbf{z}}$ is the unit vector along the surface normal and $\mathcal{C}_{(k)}$ denotes magnetic configurations with the magnetizations in the first $k \leqslant n$ $\mathrm{Cr}$ layers next to the substrate oriented parallel to the magnetization in the substrate.

\begin{tabular}{lcccccc}
\hline & $\mathcal{C}_{0}$ & $\mathcal{C}_{1}$ & $\mathcal{C}_{2}$ & $\mathcal{C}_{3}$ & $\mathcal{C}_{4}$ & $\mathcal{C}_{(k)}$ \\
\hline bcc Fe(100) & $\hat{\mathbf{z}}$ & $\hat{\mathbf{z}}$ & $\hat{\mathbf{z}}$ & $\hat{\mathbf{x}}$ & $\hat{\mathbf{x}}$ & $\hat{\mathbf{z}}$ \\
$\mathrm{Fe}$ & $\hat{\mathbf{z}}$ & $\hat{\mathbf{z}}$ & $\hat{\mathbf{z}}$ & $\hat{\mathbf{x}}$ & $\hat{\mathbf{x}}$ & $\hat{\mathbf{z}}$ \\
$\vdots$ & $\hat{\mathbf{z}}$ & $\hat{\mathbf{z}}$ & $\hat{\mathbf{z}}$ & $\hat{\mathbf{x}}$ & $\hat{\mathbf{x}}$ & $\hat{\mathbf{z}}$ \\
$\mathrm{Fe}$ & $\hat{\mathbf{z}}$ & $\hat{\mathbf{z}}$ & $\hat{\mathbf{z}}$ & $\hat{\mathbf{x}}$ & $\hat{\mathbf{x}}$ & $\hat{\mathbf{z}}$ \\
$\mathrm{Cr}$ & $\hat{\mathbf{z}}$ & $\hat{\mathbf{z}}$ & $\hat{\mathbf{z}}$ & $\hat{\mathbf{x}}$ & $\hat{\mathbf{x}}$ & 0 \\
$\vdots$ & $\hat{\mathbf{z}}$ & $\hat{\mathbf{z}}$ & $\hat{\mathbf{z}}$ & $\hat{\mathbf{x}}$ & $\hat{\mathbf{x}}$ & 0 \\
$\mathrm{Cr}$ & $\hat{\mathbf{z}}$ & $\hat{\mathbf{z}}$ & $\hat{\mathbf{z}}$ & $\hat{\mathbf{x}}$ & $\hat{\mathbf{x}}$ & 0 \\
$\mathrm{Fe}$ & $\hat{\mathbf{z}}$ & $-\hat{\mathbf{z}}$ & $\hat{\mathbf{x}}$ & $\hat{\mathbf{x}}$ & $-\hat{\mathbf{x}}$ & $\hat{\mathbf{z}}$ \\
$\vdots$ & $\hat{\mathbf{z}}$ & $-\hat{\mathbf{z}}$ & $\hat{\mathbf{x}}$ & $\hat{\mathbf{x}}$ & $-\hat{\mathbf{x}}$ & $\hat{\mathbf{z}}$ \\
$\mathrm{Fe}$ & $\hat{\mathbf{z}}$ & $-\hat{\mathbf{z}}$ & $\hat{\mathbf{x}}$ & $\hat{\mathbf{x}}$ & $-\hat{\mathbf{x}}$ & $\hat{\mathbf{z}}$ \\
Vacuum & & & & & & \\
\hline
\end{tabular}

(Weinberger et al. 1996, Blaas et al. 1999, 2001) by using $1830 \mathbf{k}_{\|}$points in the ISBZ for the occurring surface Brillouin zone integrals and an imaginary part $\delta$ to the Fermi energy of $2 \mathrm{mRyd}$. It should be noted that, clearly enough, for a restricted set of systems the $\delta \rightarrow 0$ limit can be performed (Blaas et al. 1999, 2001). However, for the large number of different cases to be shown, this is not feasible. In all calculations of electric transport, $\mathcal{P}$ and $\mathcal{A P}$ refer to the magnetic configurations $\mathcal{C}_{0}$ and $\mathcal{C}_{1}$ in table 1.

\section{§3. Results}

\subsection{Interlayer exchange coupling}

In figure 1 we show the IEC of bcc $\mathrm{Fe}(100) / \mathrm{Fe}_{6} \mathrm{Cr}_{n} \mathrm{Fe}_{6} /$ vacuum with respect to the number of $\mathrm{Cr}$ layers. $\Delta E_{\mathrm{b}}$ corresponds to the energy differences between $(a)$ the magnetic configurations $\mathcal{C}_{0}$ and $\mathcal{C}_{1}$ and $(b)$ between the magnetic configurations $\mathcal{C}_{0}$ and $\mathcal{C}_{2}$ in table 1 (see also equation (1)). Both types of coupling exhibit a short period of 2 monolayers (ML) and a long period of about 17-18 ML. As in particular the long period is of theoretical interest in figure 2 we show, for $n=17, \Delta E_{\mathrm{b}}$ as a function of the rotation angle $\Theta$ around the $y$ axis; $\Theta=90^{\circ}$ implies perpendicular alignment, $\Theta=180^{\circ}$ antiparallel alignment. As can be seen, the calculated values follow closely a $1-\cos \Theta$ behaviour, the deviations from this functional form being only marginal. Since in figures 1 and 2 the magnetic configuration $\mathcal{C}_{0}$ (ferromagnetic configuration with the orientation of the magnetization along the surface normal) serves as the reference configuration, for the particular case of $n=17$ the antiferromagnetic configuration $\mathcal{C}_{1}$ was calculated self-consistently within the LDA and used as the reference configuration. As can be seen in figure 2 at $\Theta=180^{\circ}$ the difference between these two approaches is rather small, namely about $0.6 \mathrm{meV}$, that is within the force theorem a different choice of the reference configuration causes at worst a deviation of about $6 \%$.

From figure 1, one can see that there is a ferromagnetic bias of about $11 \mathrm{meV}$ for the antiparallel IEC (5.5 $\mathrm{meV}$ for the perpendicular coupling). In order to interpret 

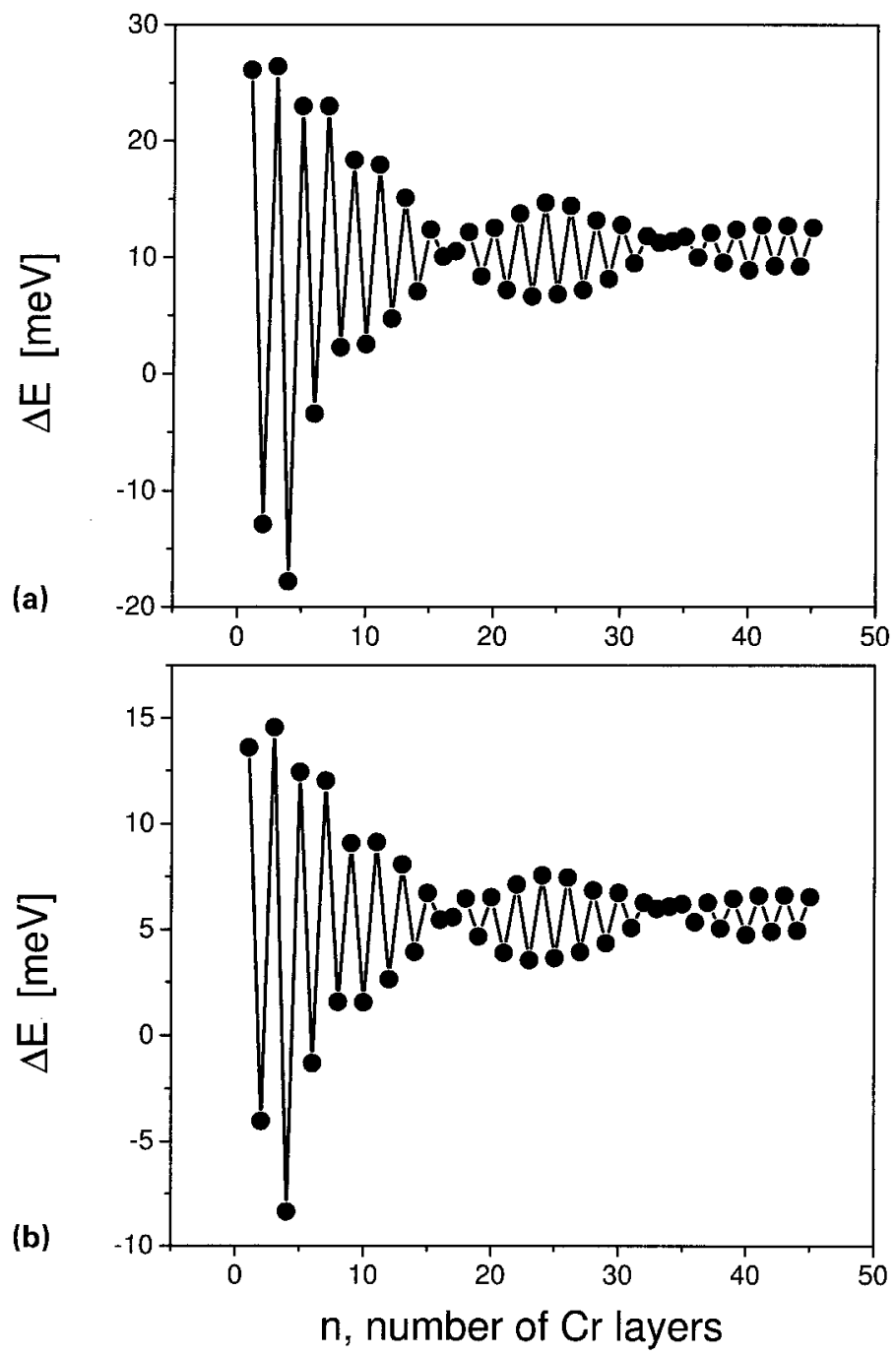

Figure 1. (a) Antiparallel and (b) perpendicular IEC in bcc $\mathrm{Fe}(100) / \mathrm{Fe}_{6} \mathrm{Cr}_{n} \mathrm{Fe}_{6} /$ vacuum, where the reference ferromagnetic configuration is $\mathcal{C}_{0}$, and the antiferromagnetic configurations are $\mathcal{C}_{1}$ and $\mathcal{C}_{2}$ (see also table 1 ).

this bias let $\Delta E_{\mathrm{b}}(r ; n ; m)$ denote the IEC for systems of the type $\mathrm{Fe}(100) / \mathrm{Fe}_{r} \mathrm{Cr}_{n} \mathrm{Fe}_{m} /$ vacuum. In principle this quantity depends weakly on the number $r$ of buffer layers considered. For a large enough $r$, however, $\Delta E_{\mathrm{b}}(r ; n ; m)$ becomes independent of $r$, that is $r$ is only a 'technical' parameter, which can be dropped. Suppose now that for a particular cap thickness $m$ the number of Cr layers becomes very large, that is

$$
\lim _{n \rightarrow N}\left[\Delta E_{b}(n ; m)\right]=\Delta E_{\mathrm{b}}(N ; m),
$$

then equation (1) implies that for $\Delta E_{\mathrm{b}}(N ; m)>0$ the ferromagnetic configuration is the ground state and consequently the antiferromagnetic configuration an excited state. It is important to note that independent of the sign of $\Delta E_{\mathrm{b}}(n ; m)$ this quantity 


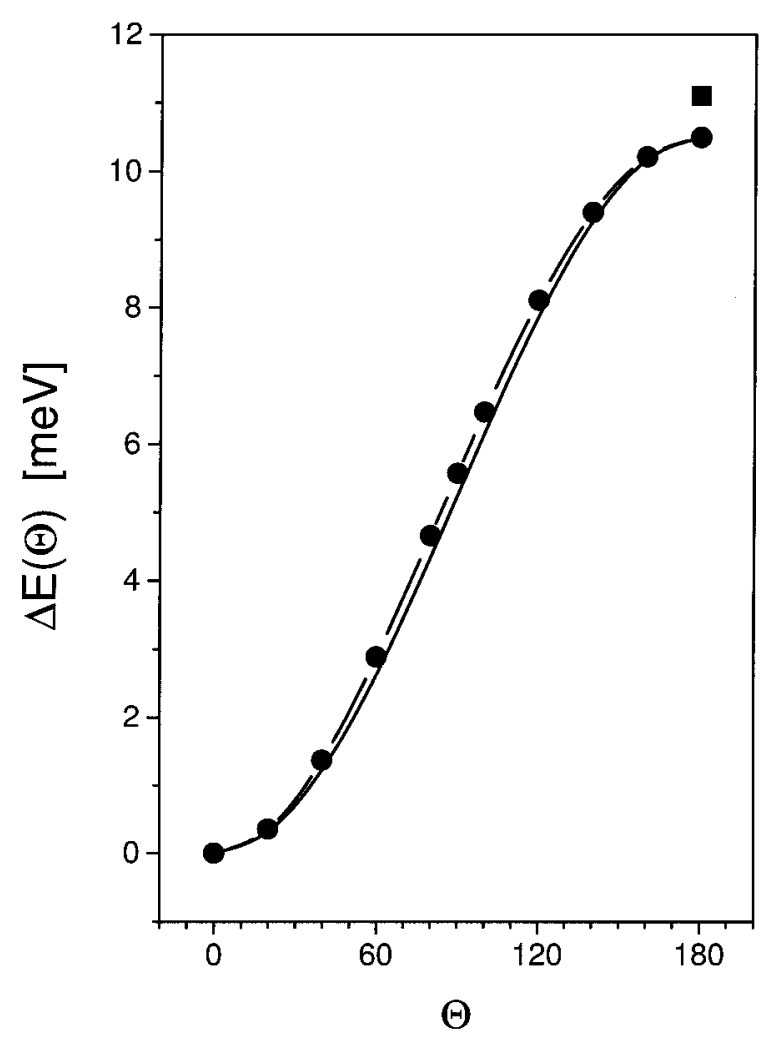

Figure 2. Angular dependence of the IEC energy in bcc $\mathrm{Fe}(100) / \mathrm{FeCr}_{17} \mathrm{Fe}_{6} /$ vacuum, where the reference ferromagnetic configuration is $C_{0}$, and the antiferromagnetic configuration $\mathcal{C}_{1}$ (see also table 1): $(\mathbf{O})$, calculated values; $(-)$, function $\Delta E(\Theta)=\Delta E(1-\cos \Theta) / 2 ;(\boldsymbol{\square})$, absolute value of the IEC energy when the (selfconsistent) antiferromagnetic configuration $\left(C_{1}\right)$ is used as the reference configuration.

always describes the energy difference between the ground state and an excited state. By defining for $n \rightarrow N$ the bias in terms of the following arithmetic mean:

$$
B(m) \sim B(N ; m)=\frac{1}{2}\left[\Delta E_{\mathrm{b}}(N ; m)+\Delta E_{\mathrm{b}}(N+1 ; m)\right],
$$

$B(m)>0$ refers to oscillations around a ferromagnetic ground state, $B(m)<0$ to oscillations around an antiferromagnetic ground state, and $B(m)=0$ to alternating ground states. For $n>7$ the oscillations in figure 1 are oscillations around a ferromagnetic ground state as $n$ becomes large.

The bias $B(n ; m)$ depends indeed on the number $m$ of Fe layers in the cap. In figure 3 we show the (antiparallel) IEC for $n=24$ and 25 as a function of the number of cap layers. It is interesting to note that an even number of $\mathrm{Cr}$ layers causes larger oscillations in the IEC with respect to the thickness of the Fe cap than an odd number of $\mathrm{Cr}$ layers does. Only by taking $m \rightarrow \infty$, that is by considering trilayers with an infinitely thick cap, can one find an 'intrinsic' value for the bias. Infinite caps, however, do not correspond to any situation encountered in an experiment. There, very often the Fe cap layer is covered by another cap of a noble metal, which in turn changes the value of the bias. In a previous paper (Kudrnovský et al. 1997), char- 




Figure 3. Interlayer exchange energy for bcc $\mathrm{Fe}(100) / \mathrm{Fe}_{6} \mathrm{Cr}_{n} \mathrm{Fe}_{m} /$ vacuum for $n=24(\bigcirc)$ and $n=25(\square)$ with respect to increasing thickness $m$ of the Fe cap, where the reference ferromagnetic configuration is $\mathcal{C}_{0}$, and the antiferromagnetic configuration $\mathcal{C}_{1}$ (see also table 1).

acteristic oscillations of the IEC in $\mathrm{Co} / \mathrm{Cu} / \mathrm{Co}$ trilayers with respect to the number of cap layers were discussed, which partially can be read off also from figure 3.

In equation (1) the interlayer exchange energy carefully was defined as the energy difference between two different magnetic configurations. The bias seen in figure 1 does indeed change by taking different antiferromagnetic configurations into account. In order to illustrate this behaviour in figure 4 the interlayer exchange energy of $\mathrm{Fe}(100) / \mathrm{Fe}_{r} \mathrm{Cr}_{n} \mathrm{Fe}_{m} /$ vacuum, $r=m=6, n=24,25$, is plotted versus the number $k$ of successive Cr layers (on top of the substrate) in which the orientation of the magnetization is parallel to the substrate (see also table 1). For a particular number $n$ of $\mathrm{Cr}$ layers this implies considering magnetic configurations with $k$ layers of $\mathrm{Cr}$ next to the substrate aligned magnetically parallel and $n-k$ subsequent layers aligned antiparallel to the orientation of the magnetization in the substrate. It should be noted that in this figure each entry refers to a different antiferromagnetic configuration. As can be seen, the bias, which is also shown in figure 4 (see equation (10)), is extremely sensitive with respect to the number of antiferromagneticall y aligned $\mathrm{Cr}$ layers and nearly vanishes in the vicinity of $k=n / 2$. Defining therefore for even (odd) $n$ a 'symmetric' magnetic configuration with the first $n / 2(\bmod (n / 2)+1) \mathrm{Cr}$ layers aligned parallel to the orientation of the magnetization in the substrate and the following $n / 2$ aligned antiparallel, the corresponding IEC oscillates now around zero with respect to $n$. As can be seen from figure 5, for this type of antiparallel coupling as well as for the correspondingly chosen perpendicular coupling, one 


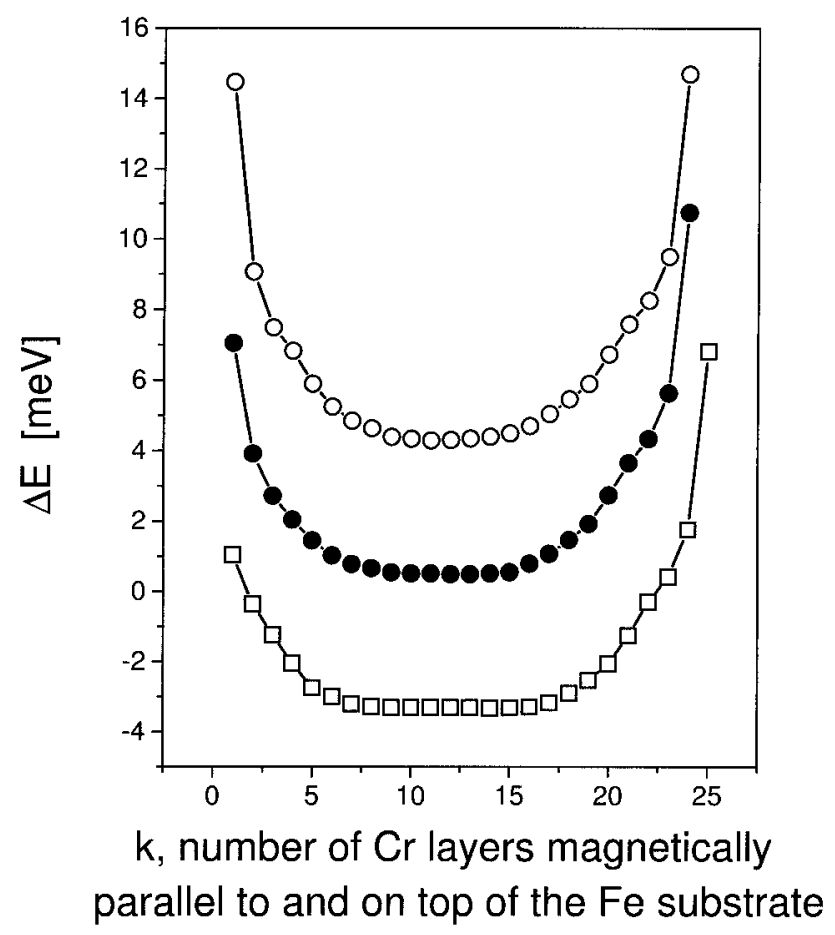

Figure 4. Interlayer exchange energy for bcc $\mathrm{Fe}(100) / \mathrm{Fe}_{6} \mathrm{Cr}_{n} \mathrm{Fe}_{6} /$ vacuum for $n=24(\bigcirc)$ and $n=25(\square)$ with respect to an increasing number $k$ of $\mathrm{Cr}$ layers on top of and aligned magnetically parallel to the Fe substrate, where the reference ferromagnetic configuration is $\mathcal{C}_{0}$, and the antiferromagnetic configurations are $\mathcal{C}_{(k)}$ (see also table 1): $(\mathbf{O})$, bias.

obtains the same features as in figure 1, namely the same short and long periods, but with a vanishing bias; figure 5 reflects the more traditional description of IEC.

In figure 6, layer-resolved contributions to the IECs with respect to the magnetic configurations $\mathcal{C}_{0}$ and $\mathcal{C}_{1}$, as well as $\mathcal{C}_{0}$ and $\mathcal{C}_{k=n / 2}$ (see equation (3)), are shown for $n=24$ and 25 . It is evident that the positive bias seen in figure 1 is mainly caused by strong positive contributions from layers close to that Fe slab in which the magnetization is reversed.

Finally it should be noted that in terms of the magnetic configurations listed in table 1 it can easily be shown that, for example,

$$
\Delta E=E\left(\mathcal{C}_{4}\right)-E\left(\mathcal{C}_{3}\right)=E\left(\mathcal{C}_{1}\right)-E\left(\mathcal{C}_{0}\right)
$$

since configuration $\mathcal{C}_{3}$ can be obtained from $\mathcal{C}_{0}$ by means of a uniform rotation by $\pi / 4$ around the $y$ axis; $\mathcal{C}_{4}$ by such a rotation from $\mathcal{C}_{1}$, that is the band energy parts of the anisotropy energy cancel out.

\subsection{Magnetic moments}

For the case of the ferromagnetic reference configuration $\left(\mathcal{C}_{0}\right.$; see table 1$)$ we show in figure 7 the magnetic moments for $\mathrm{Cr}$ thicknesses in the vicinity of the first node in the long period. While hardly any differences in the Fe moments with respect to the thickness of the $\mathrm{Cr}$ spacer can be seen, the enlarged scale for the $\mathrm{Cr}$ moments 

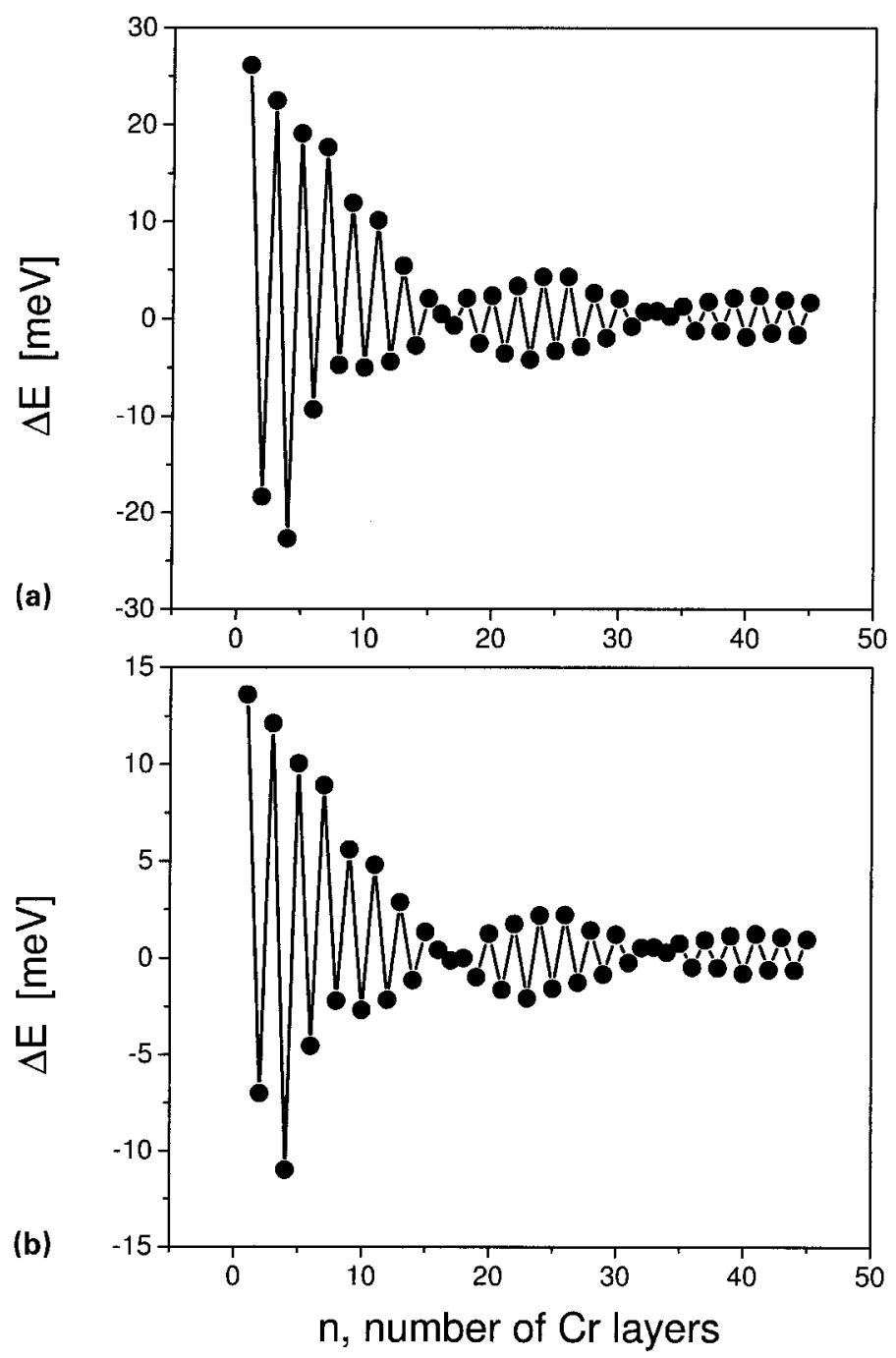

Figure 5. (a) Antiparallel and (b) perpendicular IEC in bcc $\mathrm{Fe}(100) / \mathrm{Fe}_{6} \mathrm{Cr}_{n} \mathrm{Fe}_{6} /$ vacuum, where the reference ferromagnetic configuration is $\mathcal{C}_{0}$ (see table 1 ), and the antiferromagnetic configurations are 'symmetric' (see also text).

shows clearly not only oscillations with a period of $2 \mathrm{ML}$ but also frustration effects in the case of an even number of Cr layers. For $n=17$ the magnetic moments in the self-consistently determined ferromagnetic $\left(\mathcal{C}_{0}\right)$ and in the antiferromagnetic $\left(\mathcal{C}_{1}\right)$ configurations are compared in figure 8 with each other. It is interesting to note that the symmetrically shaped oscillations of the $\mathrm{Cr}$ moments in the ferromagnetic configuration refer to the energetically more favourable state. Again the magnitude of the Fe moments remains virtually unchanged with respect to the magnetic configuration.

From figure 8 it is also clear that, when calculated self-consistently, independent of the alignment of the two Fe slabs, that is either parallel or antiparallel, the $\mathrm{Cr}$ and $\mathrm{Fe}$ layers near the $\mathrm{Fe}-\mathrm{Cr}$ interfaces always couple antiferromagnetically. 

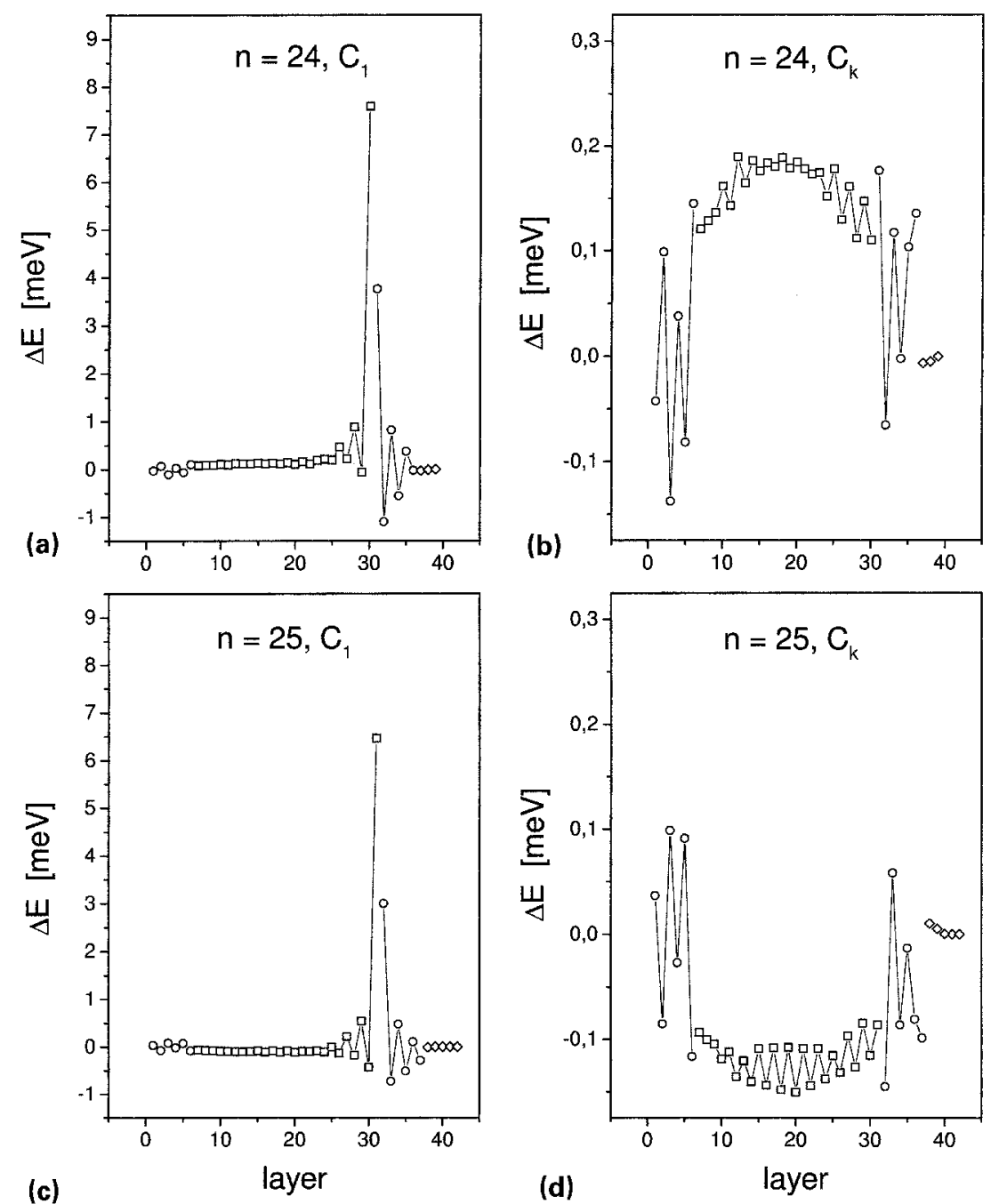

Figure 6. Layer-resolved interlayer exchange energies for bcc $\mathrm{Fe}(100) / \mathrm{Fe}_{6} \mathrm{Cr}_{n} \mathrm{Fe}_{6} /$ vacuum for (a), (b) $n=24$ and $(c),(d) n=25$ where the reference ferromagnetic configuration is $C_{0}$, and the antiferromagnetic configurations are $\mathcal{C}_{1}$ in $(a)$ and $(c)$ and $\mathcal{C}_{(k)}$ in $(b)$ and $(d)$ (see also table 1), and where the Fe substrate (origin of counting) is to the left, and vacuum to the right. $(\square)$, Cr contributions, $(\bigcirc)$, Fe contributions, $(\diamond)$, contribution from the vacuum layers.

Consequently, by using the force theorem to calculate the IEC, reversing the orientation of magnetization of the adjacent Fe slab (configuration $\mathcal{C}_{1}$ ) gives rise to the large positive energy contribution at the left $\mathrm{Fe}-\mathrm{Cr}$ interface seen in figure 6 . This naively suggests that for even $n$ (number of $\mathrm{Cr}$ layers) the configuration $\mathcal{C}_{k=n / 2}$ while for odd $n$ the configuration $\mathcal{C}_{k=(n+1) / 2}$ (see table 1) ought to be used for the most likely antiferromagnetic configuration.

Quite interesting are the $\mathrm{Cr}$ moments in rather thick $\mathrm{Cr}$ slabs. As can be seen from figure 9 for $n>40$ (in the ferromagnetic configuration $\mathcal{C}_{0}$ ) the moments not only oscillate with a period of $2 \mathrm{ML}$ but also show characteristic long periods; for an even number of $\mathrm{Cr}$ layers a period of $8 \mathrm{ML}$ is building up while for an odd number of 



Figure 7. Magnetic moments in bcc $\mathrm{Fe}(100) / \mathrm{Fe}_{6} \mathrm{Cr}_{n} \mathrm{Fe}_{6}$ /vacuum for $(a),(b) n=16,(c),(d)$ $n=17$ and $(e),(f) n=18((b),(d),(f)$ Cr moments on a blown-up scale) where the magnetic configuration is ferromagnetic with a uniform orientation of the magnetization along the surface normal $\left(\mathcal{C}_{0}\right.$ in table 1$)$, and where the Fe substrate (origin of counting) is to the left, and vacuum to the right.

Cr layers this period is doubled, that is for a large enough odd number of $\mathrm{Cr}$ layers the same long period is observed as in the IEC.

\subsection{Electric transport}

In viewing figure 10 showing the in-plane resistivities and the CIP MR in the system $\mathrm{Fe}(100) / \mathrm{Fe}_{6} \mathrm{Cr}_{n} \mathrm{Fe}_{6} /$ vacuum with respect to $n$, one has to remember that the 
ferromagnetic configuration
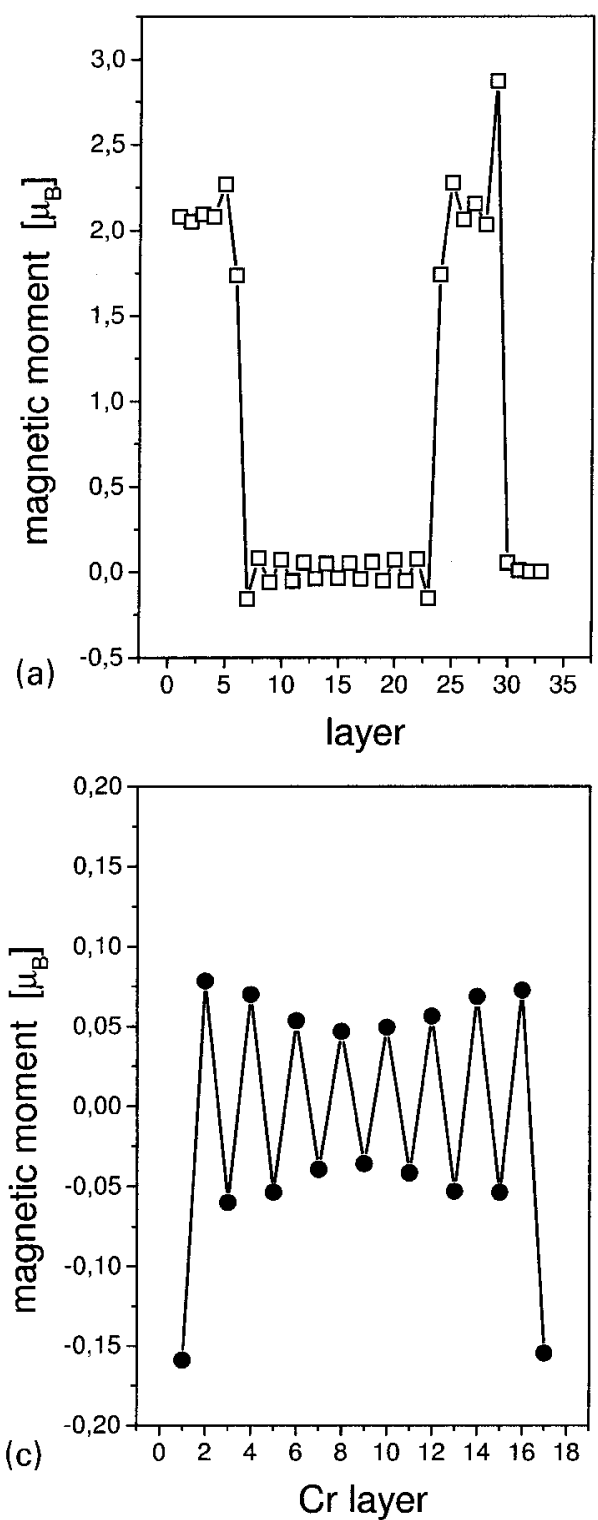

antiferromagnetic configuration


Figure 8. Magnetic moments in bcc $\mathrm{Fe}(100) / \mathrm{Fe}_{6} \mathrm{Cr}_{17} \mathrm{Fe}_{6} /$ vacuum for a (self-consistently determined) ferromagnetic $\left(\mathcal{C}_{0}\right.$, in table 1$)$ and a (self-consistently determined) antiferromagnetic configuration $\left(\mathcal{C}_{1}\right.$, in table 1$) .(c),(d) \mathrm{Cr}$ moments on a blown-up scale.

metal-vacuum interface is a so-called reflecting boundary, while the interface to the substrate usually is referred to as outgoing boundary, see, for example, the discussion given by Blaas et al. $(1999,2001)$. In general it seems that the CIP resistivities in this type of system are characterized by oscillations with a period of 3-4 ML, a feature that applies for the parallel as well as for the antiparallel configuration.

These oscillations in the resistivities cause oscillations in MR which, however, are less regular than for the resistivities. With some imagination for $5<n<17$ a period 



Figure 9. Cr magnetic moments in bcc $\mathrm{Fe}(100) / \mathrm{Fe}_{6} \mathrm{Cr}_{n} \mathrm{Fe}_{6} /$ vacuum for $(a) n=42,(b) n=44$, (c) $n=43$ and (d) $n=45$ where the magnetic configuration is ferromagnetic with the magnetization parallel to the surface normal $\left(\mathcal{C}_{0}\right.$ in table 1$)$.

of 3-4 ML seems to be present, which becomes more regular for $n>33$. At $n=13$ the MR almost vanishes. Of course, the closer in value the parallel and the antiparallel resistivities are, the more difficult it becomes to evaluate a reliable MR; for $n \geqslant 30$ the values shown have to be considered to be only tentative, although the values at $n=13$ and 30, and therefore the shape of the curve in between (extending over $17 \mathrm{ML}$ ) do appear to be part of a regular pattern. For small thicknesses of the Cr spacer $(2 \leqslant n \leqslant 8)$ the MR is quite sizeable, namely about $15 \%$.

Comparing now the MR in figure 10 with the interlayer exchange energy in figure 1 or figure 5 it is immediately evident that there is little similarity in the periods of the oscillations. At best a common $2 \mathrm{ML}$ period for small $n$ can traced. 

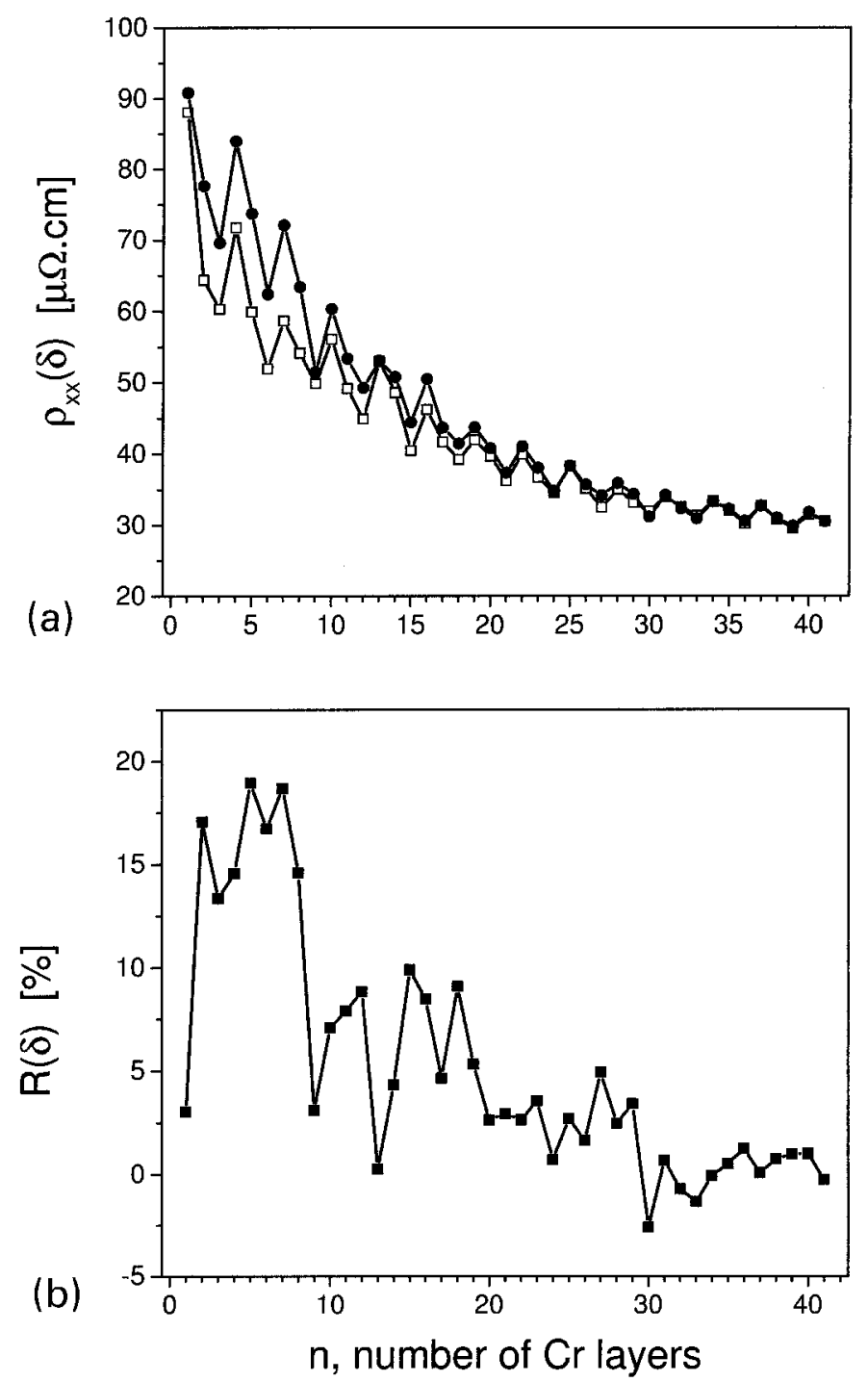

Figure 10. CIP electric transport: $(a)$ parallel $(\square)$ and antiparallel (O) in-plane resistivities and (b) MR of bcc $\mathrm{Fe}(100) / \mathrm{Fe}_{6} \mathrm{Cr}_{n} \mathrm{Fe}_{6} /$ vacuum for $n \leqslant 42$, where parallel and antiparallel correspond to the magnetic configurations $\mathcal{C}_{0}$ and $\mathcal{C}_{1}$ in table 1 . The imaginary part $\delta$ of the complex Fermi energy $\epsilon_{\mathrm{F}}+\mathrm{i} \delta$ is $2 \mathrm{mRyd}$.

It should be noted that, since in the present calculations a finite imaginary part to the Fermi energy is used, the resistivities shown in figure 10 for trilayers of the type $\mathrm{Fe}_{m} \mathrm{Cr}_{n} \mathrm{Fe}_{m}$ tend only to a constant as $n$ becomes very large:

$$
\lim _{n \rightarrow \infty}\left[\rho_{x x}\left(\mathcal{C}_{i} ; N ; \delta\right)\right]=\text { constant }, \quad N=2 m+n
$$

Only in the limit of $\delta \rightarrow 0$ does this constant reduce to zero, namely to the correct limiting behaviour. 


\section{§4. COMPARISON WITH EXPERIMENT AND OTHER THEORETICAL STUDIES}

\subsection{Interlayer exchange coupling}

At present the following experimental facts seem to be well-confirmed (Heinrich et al. 1999, 2000) for $\mathrm{Fe} / \mathrm{Cr} / \mathrm{Fe}$ trilayer systems.

(i) The exchange coupling shows a short period of about 2 ML with respect to the $\mathrm{Cr}$ thickness $d_{\mathrm{Cr}}$ and crosses to the antiferromagnetic coupling at $4 \mathrm{ML}$ of $\mathrm{Cr}$.

(ii) For $4 \leqslant d_{\mathrm{Cr}} \leqslant 12$ the samples are coupled (Krebs et al. 1989, Purcell et al. 1991, Heinrich et al. 1999, 2000) antiferromagnetically.

(iii) Opposite to the expectation that because of the $2 \mathrm{ML}$ oscillation the antiferromagnetic coupling would occur only for even numbers of $\mathrm{Cr}$ layers, independent measurements proved that this is not the case; antiferromagnetic coupling occurs at odd numbers of $\mathrm{Cr}$ layers.

(iv) The IEC depends on the thickness of the Fe cap (Pierce et al. 1999).

(v) There is a first phase slip (Unguris et al. 1991, Pierce et al. 1999) at 24$25 \mathrm{ML}(38-39 \mathrm{ML})$ of $\mathrm{Cr}$ at $310 \mathrm{~K}(550 \mathrm{~K})$ followed by phase slips at 44-45 and $64-65 \mathrm{ML}$ of $\mathrm{Cr}$ (at $310 \mathrm{~K}$ ).

It should be noted that, in order to interpret these facts properly, one has to recall that the above-cited experimental studies only give an indirect account of the IEC energy.

Comparing now these experimental findings with figures 1,3 and 5 it is evident that the present calculations reproduce the following aspects of the IEC in $\mathrm{Fe} / \mathrm{Cr} / \mathrm{Fe}$ trilayers reasonably well.

(i) There is a short period of $2 \mathrm{ML}$.

(ii) A long period of about $18 \mathrm{ML}$ of $\mathrm{Cr}$ is predicted.

(iii) The size of the IEC depends on the thickness of the Fe cap; see figure 3.

Several theoretical studies were devoted to the problem of the IEC in $\mathrm{Fe} / \mathrm{Cr} / \mathrm{Fe}$ trilayers, and in particular to the question of the long period and of the occurrence of antiferromagnetic coupling at odd numbers of $\mathrm{Cr}$ layers. Ab-initio-type supercell calculations (Hirai 1999, Hafner et al. 2000) or tight-binding (TB) model calculations (Costa et al. 1999) confirmed very nicely the short period but failed to give a sufficient account of the long period of oscillations, albeit an interpretation in terms of bulk Fermi surfaces was given (Koelling 1999). Rather recently it was reported (Niklasson et al. 1999) that by using a TB linear muffin-tin orbital surface Green's function approach for layered systems the 'spin-density wave's contribution to the IEC' oscillates with a period of two, superimposed by a long period of $20 \mathrm{ML}$ between each node or phase slip. Such spin-density wave arguments based on model calculations (Fishman 1998, Fishman and Shi 1999) were also given in other discussions of the IEC in $\mathrm{Fe} / \mathrm{Cr} / \mathrm{Fe}$ trilayers.

Usually it is difficult to compare calculated amplitudes of the IEC with those obtained by analysing the experimental data since surface roughness, chemical disorder at the interfaces (interdiffusion), but also finite-temperature (Drchal et al. 1999) effects are usually not considered in theoretical investigations. Because of these 'shortcomings' in the present paper, no attempt is made to give such a comparison to experimental amplitudes. 


\subsection{Magnetic moments}

Two facts have to be acknowledged in order to discuss magnetic moments in $\mathrm{Cr}$ related multilayer systems. First of all the $\mathrm{Cr}$ moments are sensitive not only to the actual lattice constant used but also to the explicit form of the local density functional applied (Niklasson et al. 1999, Hafner et al. 2000). However, there is also another, perhaps less well-known fact that one has to take into account. In a supercell calculation the Fermi energy has to be readjusted self-consistently for each system under investigation, whereas in approaches based on semi-infinite techniques (matching the substrate via surface Green's functions) the substrate serves as an electron reservoir, that is the Fermi energy is determined by that of the substrate. Suppose that in a supercell calculation $\epsilon_{\mathrm{F}}(n ; m)$ denotes the Fermi energy for a system characterized by a supercell containing $m$ layers of $\mathrm{Fe}$ and $n$ layers of $\mathrm{Cr}$ such as in $\ldots / \mathrm{Fe}_{m} \mathrm{Cr}_{n} \mathrm{Fe}_{m} / \ldots$ then, when either the number of $\mathrm{Cr}$ layers or the number of Fe layers becomes reasonably large $(N)$, one is confronted with the following dilemma:

$$
\epsilon_{\mathrm{F}}=\left\{\begin{array}{l}
\lim _{n \rightarrow N}\left[\epsilon_{\mathrm{F}}(n ; m)\right] \sim \epsilon_{\mathrm{F}}(C r), \\
\lim _{m \rightarrow N}\left[\epsilon_{\mathrm{F}}(n ; m)\right] \sim \epsilon_{\mathrm{F}}(F e)
\end{array}\right.
$$

Since in principle, surface Green's functions methods describe systems of the type bcc $\mathrm{Fe} / \mathrm{Cr}_{n} / \mathrm{Fe}_{m} /$ vacuum or bcc $\mathrm{Fe} / \mathrm{Cr}_{n} /$ bcc $\mathrm{Fe}$, that is true trilayer systems with a constant Fermi energy, it is sometimes difficult to make contact with supercell calculations, in particular when the number of spacer layers becomes large.

As already mentioned, the $\mathrm{Cr}$ moments in the $\mathrm{Fe} / \mathrm{Cr} / \mathrm{Fe}$ trilayer system served several workers (Fishman 1998, Fishman and Shi 1999, Hirai 1999, Niklasson et al. 1999) as a basis for discussing spin-density waves. Clearly enough the discrete Fourier transformation of the moments shown in figure 9 would give a distinct peak at $q=\pi d / 2, d$ being the interlayer distance, corresponding to a period of $2 \mathrm{ML}$, while the $q$ value of the weak peak mapping the long period of $8-9 \mathrm{ML}$ in the case of an even number of Cr layers is much too close to the trivial peak at $q=0$ to be properly resolved. However, even in the case when the number of $\mathrm{Cr}$ layers becomes rather large, a comparison with spin-density waves in bulk $\mathrm{Cr}$ would be inappropriate since in the present approach the Fermi energy (and the lattice constant) of the system still refers to bcc Fe, and since here we considered only collinear magnetic configurations.

\subsection{Magnetoresistance}

Experimental data on the GMR in $\mathrm{Fe} / \mathrm{Cr}$ multilayer systems seem to be extremely sensitive to the growth conditions (interface roughness) making not only the determination of the thickness dependence of the GMR difficult (Parkin et al. 1990, Fullerton et al. 1992), but also its actual value (Barthélémy et al. 1990, Chaiken et al. 1991, Obi et al. 1992, Omi et al. 1993, Colino et al. 1996, Schad et al. 1998) somewhat ambiguous. Typical measured resistivities at $4.2 \mathrm{~K}$ are quoted (Barthélémy et al. 1990) to be about $60 \mu \Omega \mathrm{cm}$ for a $\left[\mathrm{Fe}_{m} \mathrm{Cr}_{n}\right]$ bilayer thickness around $30 \AA$; for comparison see our calculated values in figure 10. Alloying the $\mathrm{Cr}$ spacer with $\mathrm{Fe}$ the GMR is found to decrease (Rensing et al. 1996, Ueda et al. 1996); 10\% of Fe is sufficient (Ueda et al. 1996) to wipe out the effect essentially.

Quite clearly, in order to increase the GMR effect, in most experimental studies repetitions of double layers of the type $\left[\mathrm{Fe}_{m} \mathrm{Cr}_{n}\right]_{r}$ were considered (for example 
Baibich et al. (1988)). In one particular case study (Barnas et al. 1990) an increase in the GMR from $4 \%$ at $r=1$ to $14 \%$ at $r=4$ was found. Unfortunately when measuring repetitions the thickness of the Fe layer also enters as a system parameter and has to be varied (Petroff et al. 1991, Miyazaki et al. 1992, Hahn et al. 1994, Schad et al. 1996).

In early experiments (Potter et al. 1994) on the MR at $4.2 \mathrm{~K}$ of $\mathrm{Fe} / \mathrm{Cr} / \mathrm{Fe}$ trilayers a $2 \mathrm{ML}$ oscillation was observed as the $\mathrm{Cr}$ thickness was varied between 4 and approximately $14 \mathrm{ML}$; four peaks were recorded in the GMR (at 4, 6, approximately 8 , and $10 \mathrm{ML}$ ) which varied between 0 and $2.5 \%$. Magneto-optical Kerr angle measurements in the same regime of $\mathrm{Cr}$ thicknesses also showed $2 \mathrm{ML}$ oscillations; many researchers inferred that the oscillations in the GMR and the IEC had a common origin and are interrelated. By comparing the GMR data of Potter et al. (1994) with our results in figure $10(b)$, one can see that in the quoted regime of $\mathrm{Cr}$ thicknesses $(4 \leqslant n \leqslant 14)$ the theoretical calculations show only three distinct peaks, namely a peak at $5 \mathrm{ML}$, a peak at $7 \mathrm{ML}$ and a rather broad peak (double peak) between 9 and $11 \mathrm{ML}$, and the GMR varies between 0 and about $20 \%$. In the theoretical calculations the $2 \mathrm{ML}$ oscillation seen in experiment seems therefore to be shifted by about $1 \mathrm{ML}$. By using repetitions of $\left[\mathrm{Fe}_{m} \mathrm{Cr}_{n}\right]$ bilayers, peaks in the GMR have been found (Parkin et al. 1990, Gijs and Okada 1992a) at about 10, 25 and $45 \AA$; however, this has nothing in common with the long-period oscillations in the IEC.

Considering the broad range of reported resistivities we did not aim to calculate the actual resistivities of these trilayers; this requires knowledge of the impurity distribution and interdiffusion profiles in these structures. Therefore the use of a finite $\delta$, which introduces an artificial distribution of the scattering, does not seem to be a serious drawback if we are primarily interested in calculating the GMR ratios.

Besides some applications (Gijs and Okada 1992b, 1993) of the so-called quantum model (Levy et al. 1990, Zhang et al. 1992), Kubo-type approaches have been employed (Tsymbal and Pettifor 1996, Mathon 1997) in TB parametrized schemes, however, making use of superlattices, that is of periodicity along the growth direction of the multilayer system. For periodic $\mathrm{Fe} / \mathrm{Cr}$ multilayers, for example sheet resistances (in the CPP geometry) of about $5 \times 10^{-15} \Omega \mathrm{m}^{2}$ are reported (Mathon 1997) that lead to a CPP MR of about $8 \%$ for 100 repetitions. An ab-initio-type calculation (Mertig et al. 1995) based on the Boltzmann equation in the relaxation time approximation and assuming periodicity along the surface normal did in fact give a $2 \mathrm{ML}$ oscillation around about $6 \%$ for the CIP MR with respect to the $\mathrm{Cr}$ thickness with peaks in the CIP MR at 5, 7, 9, 11 and $13 \mathrm{ML}$ (the range investigated by Potter et al. (1994)). The corresponding CPP MR did not show such oscillations and however, was much higher in value (about $25 \%$ ).

\section{$\S 5$. CONCLUSION}

In the present paper, ab-initio calculations of the IEC and of the (CIP) electric transport properties in $\mathrm{Fe} / \mathrm{Cr} / \mathrm{Fe}$ trilayers are based on a uniformly applied, fully relativistic spin-polarized approach. The results obtained seem to fit reasonably well to the by now commonly accepted experimental facts. However, there still remain two basic questions connected with the present calculations of the IEC, namely the actual size of the bias of the IEC seen in experiment and the problem of collinearity of the $\mathrm{Cr}$ moments. Quite clearly this bias is partly determined 
(i) by the form of the density functional parameters used,

(ii) by the lattice spacing,

(iii) by the conceptual constraints to the Fermi energy,

(iv) by errors inherent to the force theorem, and

(v) in particular by the antiferromagnetic configuration chosen.

Since it is virtually impossible to vary all relevant parameters and, in particular, to obtain sufficiently accurate total energy differences well converged with respect to the occurring Brillouin zone integrations, for the time being one merely can draw attention to these drawbacks.

Non-collinearity of the $\mathrm{Cr}$ moments not only can reduce the bias but also quite likely can modulate the periods of oscillations. Non-collinearity in this context means that within the $\mathrm{Cr}$ layers the moments are still aligned collinearly, the direction of alignment being, however, different in different Cr layers (Fishman 1999). Non-collinearity has to be taken into account also when making contact to spindensity wave theories.

In terms of electric transport the experimental data are perhaps less decisive since the sample characterization is still not sufficiently accurate to enable a comparison on an absolute scale. For this reason the approach used in here, namely calculating resistivities for a finite imaginary part of the Fermi energy seems to be sufficient to discuss basic qualitative features and to give a semiquantitative account of the experimental data. Quite clearly, as soon as there are measurements available for well-characterized samples, and corresponding resistivities (and not only the MR) are reported, it will be beneficial to vary the antiferromagnetic configuration and to continue analytically the calculated resistivities to the real axis.

Finally it should be noted that the widespread belief in a common cause for the peaks in the GMR and the oscillations in the IEC clearly does not follow from the experimental evidence nor from the present calculations.

\section{ACKNOWLEDGEMENTS}

This paper resulted from a collaboration partially funded by the TMR network on 'Ab-initio calculations of magnetic properties of surfaces, interfaces, and multilayers' (Contract EMRX-CT96-0089) and the Research and Technological Cooperation between Austria and Hungary (OMFB-BMAA) (contract A-35/98). Financial support was provided also by the Center of Computational Materials Science (contract GZ 45.451), the Austrian Science Foundation (contracts P11626, P12352 and T27-TPH), and the Hungarian National Science Foundation (Contracts OTKA T030240 and T022609). This work was supported by the Defense Advanced Research Projects Agency and Office of Naval Research under grant N00014-96-1 1207.

\section{REFERENCES}

Baibich, M. N., Broto, J. M., Fert, A., Dau, F. N. V., Petroff, F., Etienne, P., Creuzet, G., Friedrich, A. and Chazelas, J., 1988, Phys. Rev. Lett., 61, 2472.

Barnas, J., Fuss, A., Camley, R. E., Grünberg, P., and Zinn, W., 1990, Phys. Rev. B, 42, 8110.

Barthélémy, A., Fert, A., Baibich, M. N., Hadjoudj, S., Petroff, F., Etienne, P., Cabanel, R., Lequien, S., Dau, F. N. V., and Creuzet, G., 1990, J. appl. Phys., 67, 5908.

Baumgart, P., Gurney, B. A., Wilhoit, D. R., Nguyen, T., Dieny, B., and Speriosu, V. S., 1991, J. appl. Phys., 69, 4792. 
Blaas, C., Weinberger, P., Szunyogh, L., Levy, P. M., and Sommers, C., 1999, Phys. Rev. B, 60, 492.

Blats, C., Weinberger, P., Szunyogh, L., Sommers, C., and Levy, P. M., 2001, Phys. Rev. B, 63, 224408.

Chaiken, A., Tritt, T. M., Gillespie, F. J., Krebs, J. J., Lubitz, P., Harford, M. Z., and Prinz, G. A., 1991, J. appl. Phys., 69, 4798.

Colino, J. M., Schuler, I. K., Korenivski, V., and Rao, K. V., 1996, Phys. Rev. B, 54, 13030.

Costa, A. T., D’Albuquerque e Castro, J., and Muniz, R. B., 1999, Phys. Rev. B, 59, 11424.

Drchal, V., Kudrnovský, J., Bruno, P., Dederichs, P. H., and Weinberger, P., 1999, Phys. Rev. B, 60, 9588.

Fishman, R. S., 1998, Phys. Rev. B, 57, 10 284; 1999, ibid., 59, 13849.

Fishman, R. S., and Shi, Z. P., 1999, Phys. Rev. B, 59, 13849.

Fullerton, E. E., Kelly, D. M., Schuller, I. K., and Bruynseraede, Y., 1992, Phys. Rev. Lett., 68, 859.

Gijs, M. A. M., Giesbers, J. B., Johnson, M. T., De Steege, J. B. F., and Janssen, H. H. J. M., 1994, J. appl. Phys., 75, 6709.

Gijs, M. A. M., Lenczowski, S. K. J., and Giesberg, J. B., 1993, Phys. Rev. Lett., 70, 3343.

Giss, M. A. M., and Okada, M., 1992a, J. Magn. magn. Mater., 113, 105; 1992b, Phys. Rev. B, 46, 2908; 1993, J. Magn. magn. Mater., 121, 71.

Hafner, R., Spis̃ák, D., Lorenz, R., and Hafner, J., 2000, J. appl. Phys., 87, 5756.

Hahn, W., Loewenhaupt, M., Felcher, G. P., Huang, Y. Y., and Parkin, S. S. P., 1994, J. appl. Phys., 75, 3564.

Heinrich, B., Cochran, J. F., Monchesky, T., and Urban, R., 1999, Phys. Rev. B, 59, 14 520; 2000, J. appl. Phys., 87, 5449.

Hirai, K., 1999, Phys. Rev. B, 59, R6612.

Ho, E. M., and Petford-Long, A. K., 1996, J. Magn. magn. Mater., 156, 65.

Ho, E. M., Petford-Long, A. K., and Cerenzo, A., 1999, J. Magn. magn. Mater., 192, 431.

Hosoito, N., Mibu, K., Ono, T., Shinjo, T., and Endoh, Y., 1993, J. Magn. magn. Mater., 126, 255.

Jacob, M., Reiss, G., Brückl, H., and Hoffman, H., 1992, Phys. Rev. B, 46, 11208.

Kamijo, A., and Igarashi, H., 1992, J. appl. Phys., 72, 3497.

Koelling, D. D., 1999, Phys. Rev. B, 59, 6351.

Krebs, J. J., Lubitz, P., Chaiken, A., and Prinz, G. A., 1989, Phys. Rev. Lett., 63, 1645.

Krebs, J. J., Lubitz, P., Haiken, A., and Prinz, G. A., 1991, J. appl. Phys., 69, 4795.

Kudrnovský, J., Drchal, V., Bruno, P., Turek, I., and Weinberger, P., 1997, Phys. Rev. B, 56, 8919.

Levy, P. M., Zhang, S., and Ferta, A., 1990, Phys. Rev. Lett., 65, 1643.

Levy, S. Z. P. M., 1991, J. appl. Phys., 69, 4786.

Mathon, J., 1997, Phys. Rev. B, 55, 960.

Mertig, I., Zahn, P., Richter, M., Eschrig, H., Zeller, R., and Dederichs, P. H., 1995, J. Magn. magn. Mater., 151, 363.

Mirbt, S., Niklasson, A. M. N., Johansson, B., and Skriver, H. L., 1996, Phys. Rev. B, $\mathbf{5 4 ,} 6382$.

Miyazaki, T., Kubota, H., and Ishio, S., 1992, J. Magn. magn. Mater., 103, 13.

Niklasson, A. M. N., Johansson, B., and Nordström, L., 1999, Phys. Rev. Lett., 82, 4544.

Obi, Y., Takanashi, K., Mitani, Y., Tsuda, N., and Fujimori, H., 1992, J. appl. Phys., 104-107, 1747.

Omi, G., Uwatoko, Y., Obi, Y., Takanashi, K., and Fujimori, H., 1993, J. Magn. magn. Mater., 126, 513.

Parkin, S. S. P., More, N., and Roche, K. P., 1990, Phys. Rev. Lett., 64, 2304.

Parkin, S. S. P., and York, B. R., 1993, Appl. Phys. Lett., 62, 842.

Petroff, F., Barthélémy, A., Hamzić, A., Fert, A., Etienne, P., Lequien, S., and CreuZET, G., 1991, J. Magn. magn. Mater., 93, 95.

Pierce, D. T., Unguris, J., Celotta, R. J., and Stiles, M. D., 1999, J. Magn. magn. Mater., 200, 290. 
Potter, C. D., Schad, R., Belien, P., Verbanck, G., Moshchalkov, V. V., BruynserAede, Y., Schäfer, M., Schäfer, R., and Grünberg, P., 1994, Phys. Rev. B, 49, 16055.

Purcell, S. T., Folkerts, W., Johnson, M. T., McGee, N. W. E., J Ager, K., De Steege, J. B. F., Zeper, W. B., Hoving, W., and Grünber g, P., 1991, Phys. Rev. Lett., 67, 903.

Rensing, N. M., Clemens, B. M., and Williamson, D. L., 1996, J. appl. Phys., 79, 7757.

Schad, R., Barnas, J., Belien, P., Verbanck, G., Potter, C. D., Fischer, H., Lefebvre, S., Bessiere, M., Moshchalkov, V. A., and Bruynser aede, Y., 1996a, J. Magn. magn. Mater., 156, 339.

Schad, R., Belien, P., Barnas, J., Verbanck, G., Potter, C. D., Glayszewski, G., Moshchalkov, V. V., and Bruynseraede, Y., 1996b, J. Magn. magn. Mater., 156, 341.

Schad, R., Belien, P., Verbanck, G., Potter, C. D., Fischer, H., Lefebvre, S., Bessiere, M., Moshchalkov, V. V., and Bruynseraede, Y., 1998, Phys. Rev. B, 57, 13692.

Schad, R. Potter, C. D., Belien, P., Verbanck, G., Dekoster, J., Langouche, G., Moshchalkov, V. V., and Bruynseraede, Y., 1995, J. Magn. magn. Mater., 148, 331.

Schad, R., Potter, D. D., Belien, P., Verbanck, G., Moshchalkov, V. V., Bruynseraede, Y., Lenczowski, S. K. J., and Giesberg, J. B., 1994a, Appl. Phys. Lett., 64, 3500.

Schad, R., Potter, C. D., Belien, P., Verbanck, G., Moshchalkov, V. V., BruynserAede, Y., Schäfer, M., Schäfer, R., and Grünberg, P., 1994b, J. appl. Phys., 76, 6604.

Stoeffler, D., and Gautier, F., 1993, J. Magn. magn. Mater., 121, 359.

Szunyogh, L., Újfalussy, B., and Weinberger, P., 1995, Phys. Rev. B, 51, 9552.

Tsymbal, E., and Pettifor, D. G., 1996, Phys. Rev. B, 54, 15314.

Ueda, Y., Ikeda, S., Hama, S., and Yamada, A., 1996, J. Magn. magn. Mater., 156, 353.

Unguris, J., Celotta, R. J., and Pierce, D. T., 1991, Phys. Rev. Lett., 67, 140.

Ustinov, V. V., Bebenin, N. G., Romashev, L. N., Minin, V. I., Milyaev, M. A., Del, A. R., and Semerikov, A. V., 1996a, Phys. Rev. B, 54, 15958.

Ustinov, V. V., Romanshev, L. N., Bebenin, N. G., Minin, V. I., Kazantsev, V. A., and Milyaev, M. A., 1996b, J. Magn. magn. Mater., 156, 227.

Vosko, S. H., Wilk, L., and Nusair, M., 1980, Can. J. Phys., 58, 1200.

Weinberger, P., 1997, Phil. Mag. B, 75, 509.

Weinberger, P., Levy, P. M., Banhart, J., Szunyogh, L., and Újfalussy, B., 1996, J. Phys.: condens. Matter, 8, 7677.

Weinberger, P., and Szunyogh, L., 2000, Comput. Mater. Sci., 17, 414. 